\title{
Restrictions on Information Transfer in Quantum Measurements
}

\author{
S.N.Mayburov \\ Lebedev Inst. of Physics \\ Leninsky Prospect 53, Moscow, Russia, 117924 \\ E-mail : mayburov@sci.lebedev.ru
}

\begin{abstract}
Information-Theoretical restrictions on the information transfer in quantum measurements are studied for practical systems. For the measurement of quantum system $S$ by detector D, registrated and processed by information system $O$, such restrictions are described by formalism of inference maps in Hilbert space. The final $O$ restricted states $\xi^{O}$ calculated from the agreement with Shrödinger $S, \mathrm{D}, O$ dynamics. It's shown that the principal information losses from $S$ to $O$ stipulate the stochasticity of measurement outcomes; consequently $\xi^{O}$ describes the random 'pointer' outcomes $q_{j}$ observed by $O$ in the individual events.
\end{abstract}

Talk given on 'Quantum Information and Computations' workshop, Torino, May 2006

\section{Introduction}

Despite its significant achievements, Measurements Theory of QM still contains some open questions concerned with its internal consistency ${ }^{1,2,3}$. The most famous and oldest of them is the State Collapse or Objectification Problem, but there are also others more subtle and less popular $^{4,5,6}$. In this paper the Problem of Quantum Measurement are studied mainly within the framework of Information Theory, with complete account of Quantum Dynamics results for Measuring System (MS. Really, the measurement of some system $S$ is also the transfer of information from $S$ to the information system $O$ (Observer), which proceses and memorizes it. Therefore the possible restrictions on the information transfer from $S$ to $O$ can influence the effects observed in the measurements ${ }^{7}$. In our previous paper it was shown that this restrictions are principally important and,in particular, result in the unavoidable stochasticity in the outcomes of measurements ${ }^{8}$. Our calculations of this effects exploited QM formalism of Observable Algebra - $C^{*}$-algebra which is most general and deep mathematical QM formulation ${ }^{4}$. 
However this formalism is rather complicated and abstract, so in this paper the same results are obtained by means of standard Schrödinger QM formalism; it permits also to analyze the fundamental QM aspects more easily. The modified formalism of inference maps in Hilbert space ${ }^{5}$ is applied here for the calculations of information transfer from $\mathrm{S}$ to $O$. In our approach the evoultion of all objects, including macroscopic ones, is described by the quantum state $\rho(t)$ which obeys to Schrödinger-Lioville equation in arbitrary reference frame (RF). In this framework QM eigentstate axiom(EA) is generic: for any $S$ observable $G$ there at least two $S$ states $\left\{\rho_{i}\right\}$ for which S posess the different real properties ${ }^{1}-g_{i}$ which are $G$ eigenvalues. Hence EA predicts unambigously the results of $G$ measurements for its eigenstates $\rho_{i}$ in the individual events of measurement. Remind ${ }^{4}$ that in general an arbitrary $\rho$ will be $G$ eigenstate with eigenvalue $g_{a}$ : $G \rho \rightarrow g_{a}$, if $\bar{G}^{l}=(\bar{G})^{l}=g_{a}^{l}$ for any natural $l>0$. As shown below, the set of such $G$ eigenstates $\left\{\rho_{i}\right\}$ contitutes for given $S$ the 'information' basis, and it permits to find the properies of arbitrary state $\rho^{\prime}$ from its comparison with $\left\{\rho_{i}\right\}$. We shall argue also that EA together with some Information-Theoretical constraints permit to formulate the consistent measurement formalism, and to derive, in fact, QM Reduction Postulate from other well-known QM postulates. Further details concerning with the inference maps, systems self-description, etc., can be found elsewhere ${ }^{6,8}$.

\section{Model of Measurements}

Here our measurement model will be described and some aspects of Measurement Theory essential for our approach discussed. The measuring system (MS) of our model consists of the studied system $\mathrm{S}$, detector $\mathrm{D}$ and the information system $O$ which isn't regarded in this chapter as a quantum object in a strict sense (it will be done in chap. 3). Here S,D system is described in fact by Zurek ansatz ${ }^{10}$, quite popular for the discussion of QM foundations. In our approach $\mathrm{S}$ is the particle with spin $\frac{1}{2}$ and the measurement of its projection $S_{z}$ is regarded, its $u, d$ eigenstates denoted $\left|s_{i}\right\rangle ; i=1,2$. Analogously to $\mathrm{S}$ in our model $\mathrm{D}$ state is also described by Dirac vector $|D\rangle$ in two-dimensional Hilbert space $\mathcal{H}_{\mathcal{D}}$. Its basis constitutes the orthogonal states $\left|D_{1,2}\right\rangle$, which are the eigenstates of $Q$ 'pointer' observable with eigenvalues $q_{1,2}$. The initial $\mathrm{D}$ state is: $\left|D_{0}\right\rangle=\frac{1}{\sqrt{2}}\left(\left|D_{1}\right\rangle+\left|D_{2}\right\rangle\right)$, the measured S state: $\psi_{s}=a_{1}\left|s_{1}\right\rangle+a_{2}\left|s_{2}\right\rangle$. S,D interaction $\hat{H}_{S, D}$ starts at $t_{0}$ and finishes effectively at some $t_{1}$; for Zurek Hamiltonian $H_{S, D}$ it would result in S,D final state:

$$
\Psi_{S, D}=\sum a_{i}\left|s_{i}\right\rangle\left|D_{i}\right\rangle
$$

relative to $O$ or any other RF. It turns out that $\bar{Q}=\left|a_{1}\right|^{2}-\left|a_{2}\right|^{2}$, so $D$ performs $S_{z}$ measurement of first kind ${ }^{1}$. The measurement of incoming S 'test' mixture with the same $\bar{S}_{z}$ will be regarded here also for the comparison. Such $\mathrm{S}$ ensemble represented by the gemenge ${ }^{1} W^{s}=\left\{\left|s_{i}\right\rangle, P_{i}=\right.$ $\left.\left|a_{i}\right|^{2}\right\}$, where $P_{i}$ is the probability of individual state $\left|s_{i}\right\rangle$ appearance, it described statistically by the density matrix:

$$
\rho_{s}^{m}=\sum_{i}\left|a_{i}\right|^{2}\left|s_{i}\right\rangle\left\langle s_{i}\right|
$$

At $t>t_{1} \mathrm{D}$ presumably interacts with $O$ and thus transfers to it some information. At this stage $O$ isn't regarded as quantum object exactly, rather we shall use more loose setting, assuming only that $O$ is macroscopic object which doesn't violate QM laws. It's supposedly true for human observer also, and below some terms characteristic for human perception will be used for simplicity.

Consider first the measurement of $\mathrm{S}$ eigenstate $\left|s_{1,2}\right\rangle$, it results in $\Psi^{1,2}=\left|s_{1,2}\right\rangle\left|D_{1,2}\right\rangle$ factorized S,D state, for final $\left|D_{1,2}\right\rangle$ its eigenvalue $q_{1,2}-\mathrm{D}$ pointer position is $\mathrm{D}$ real property ${ }^{1}$, 
corresponding to the orthogonal projector $P_{1,2}^{D}$. Hence the difference between this $\mathrm{D}$ states is objective or Boolean Difference $^{3}$ (BD). In general it means that for two states $\varphi_{1,2}$ in given $O$ $\mathrm{RF}$, their difference is equivalent to the distinction between the logical operands $Y e s / N o$, or that's the same between the values $1 / 0$ of some discrete parameter $l_{g}$ available for $O$. However in QM such parameters are related to Observables - Hermitian Operators, so not for all $\varphi_{1,2}$ states BD would hold, and it will have the important consequences in our theory. In the regarded case $\mathrm{BD}$ implicates also that in a given event $n O$ supposedly percepts $\left|D_{1,2}\right\rangle$ states as the information patterns ${ }^{6}$ (IP):

$$
J^{O}(n)=J_{1,2}^{O}=q_{1,2}
$$

- $O$ 'impressions' (see below). Remind that in general in given event $n \operatorname{IP} J(n)=\left\{e_{1}(n), \ldots, e_{l}(n)\right\}$ is the set of numerical parameters values available for observation in given $\mathrm{RF}^{6}$.

Now let's regard the possible measurement outcome for the case when $a_{1,2} \neq 0$, i.e. is $s_{i}$ superposition of (1). 'Pedestrian Interpretation ${ }^{2}(\mathrm{PI})$ claims that in this case $O$ should percept S,D state $\Psi_{S, D}$ as the superposition - the simultaneous coexistence of two IP $q_{1}$ and $q_{2}$, or at least should differ it from $\left|D_{1,2}\right\rangle$. Yet the situation isn't so simple and doesn't favor such prompt jump to conclusions. Really, given PI is correct, $O$ should distinguish in a single event $\Psi_{S, D}$, or that's the same its partial D state $R_{d}$, from each $\left|D_{i}\right\rangle$ which $O$ percepts as $J_{1,2}^{O}$. Hence for $O$ their relation should be also expressed as $Y e s / N o$ - or $1 / 0$, i.e. to be characterized at least by one D parameter $g^{D}$ which values are different for this cases. However, to confirm this conclusion one should look for the suitable D observable $G^{D}$ for which $g^{D}$ are eigenvalues, because, as was noticed above, in QM all feasible parameters correspond to QM observables. The possibility that such observable is $Q, P_{i}^{D}$ or their linear form is excluded beforehand, because $Q$ has only two eigenvalues $q_{1,2}$ occupied by $\left|D_{1,2}\right\rangle$. The simple check shows that any other $\mathrm{D}$ observable also fails to describe $g^{D}$, and $R_{d}$ doesn't have the projector on $\mathcal{H}_{\mathcal{D}}$ (the role of joint S,D observables regarded below). It can be shown that the parameter $g^{D}$ with such properties responds to nonlinear operator in $\mathcal{H}_{\mathcal{D}}$ only, so its observation is incompatible with standard QM formalism. Hence it's impossible for $O$ in this approach to distinguish $\left|D_{i}\right\rangle$ from $R_{d}$ i.e. from $\Psi_{S, D}$ in a single event. Consequently PI results in the contradiction in this simplified framework, the analogous conclusion was made by Wigner ${ }^{9}$. One can regard it as the reason to introduce Reduction Postulate in QM, however before any conclusions will be done, it's instructive to consider the possible influence of $O$ quantum properties on the measurement outcome.

\section{Quantum Measurement from Inside}

Now the information system $O$ will be regarded as the quantum object described by pure state relative to some other $\mathrm{RF} O^{\prime}$. We choose the same $O$ internal structure as for D: i.e. its pure state is a vector in two-dimensional Hilbert space $\mathcal{H}_{\mathcal{O}}$. Analogously to D $O$ initial state $\left|O_{0}\right\rangle=$ $\frac{\left|O_{1}\right\rangle+\left|O_{2}\right\rangle}{\sqrt{2}}$, where $\left|O_{i}\right\rangle$ are eigenstates of $O$ 'internal pointer' observable $Q_{O}$ with eigenvalues $q_{i}^{O}$. For suitable D, $O$ Hamiltonian $H_{D, O}$ one can obtain at some $t>t_{1}$ :

$$
\Psi_{M S}=\sum a_{i}\left|s_{i}\right\rangle\left|D_{i}\right\rangle\left|O_{i}\right\rangle
$$

relative to some external RF $O^{\prime}$. At easy to see $\mathrm{D}$ states only double $\mathrm{S}$ states for this ansatz, so below $\mathrm{D}$ will be dropped for the simplicity.

In Information Theory the measurement of an arbitrary $S^{\prime}$ by another system $O^{I}$ is the mapping of $S^{\prime}$ states set $N_{S}$ to the set $N_{O}$ of $O^{I}$ states $^{6}$. In general case which is generic for $\mathrm{QM}, O^{I}$ can be considered as the subsystem of large system $S_{T}=S^{\prime}+O^{I}$ with the states set 
$N_{T}$, i.e. can be described by formalism of Systems Self-description ${ }^{7}$. The inference map $M_{T}$ of $S_{T}$ state to $N_{O}$ - set of $O^{I}$ states, defines the corresponding $O^{I}$ state $R_{O}$ called $S_{T}$ restricted state. In this formalism called 'measurement from inside' $R_{O}$ contains maximal information on $S_{T}$ transferred to $O^{I}$. The important property of inference map $M_{T}$ is formulated by Breuer Theorem: if for two arbitrary $S_{T}$ states $\Gamma_{S}, \Gamma_{S}^{\prime}$ their restricted states $R, R^{\prime}$ coincide, then for $O^{I}$ this $S_{T}$ states are indistinguishable ${ }^{5}$. In classical case the origin of this effect is obvious: $O^{I}$ has less degrees of freedom than $S_{T}$ and hence can't discriminate all possible $S_{T}$ states. In quantum case the entanglement and nonlocality play the additional important part in this effect. Despite that $R_{O}$ are incomplete $S_{T}$ states, they are the real physical states for $O^{I}$ observer - 'the states in their own right', as Breuer characterizes them.

Regarded relations between $S^{\prime}, O^{I}, S_{T}$ states are applicable to regarded MS model which can be treated as MS measurement from inside. However Schrödinger QM formalism by itself doesn't permit to derive the inference map $M_{T}\left(S_{T} \rightarrow O^{I}\right)$ in quantum case directly. Breuer proposed phenomenologically that for arbitrary $S_{T}$ its restricted state is equal to the partial trace of $S_{T}$ individual state over $S^{\prime}$, i.e. $R_{O}$ is $S_{T}$ partial state in $O^{I}$. Then in our case for MS pure state $\Psi_{M S}$ of (3) it gives:

$$
R_{O}=\operatorname{Tr}_{s} \rho_{M S}^{p}=\sum\left|a_{i}\right|^{2}\left|O_{i}\right\rangle\left\langle O_{i}\right|
$$

Obviously such ansatz excludes beforehand any kind of probabilistic $R_{O}$ behavior, and this is natural for Schrödinger formalism. For MS mixture induced by incoming $W^{s}$ ensemble of (2) the individual MS state differs from event to event:

$$
\varsigma^{M S}(n)=\left|O_{l}\right\rangle\left\langle O_{l}|| s_{l}\right\rangle\left\langle s_{l}\right|
$$

where the random $l(n)$ frequencies stipulated by the probabilistic distribution $P_{l}$. In Breuer ansatz $O$ restricted state for this ensemble is also stochastic $R_{O}^{\operatorname{mix}}(n)=\xi_{i}^{O}$, where $\xi_{i}^{O}=\left|O_{i}\right\rangle\left\langle O_{i}\right|$ appears with corresponding probability $P_{i}$. In this case the resulting $q_{i}^{O}$ are the objective $O$ properties, hence $O$ percepts the incoming $\left|s_{i}\right\rangle$ states as IP $J^{O}(n)=q_{i}^{O}$. In general all IP properties described in chap. 2, are fulfilled also for $O$ in self-description formalism ${ }^{8} . R_{O}^{\text {mix }}(n)$ differs from $R_{O}$ in any event $n$, so for the restricted individual states the main condition of cited theorem is violated. From that Breuer concluded that $O$ can discriminate the individual pure/mixed MS states 'from inside', and hence $O$ wouldn't observe the collapse of pure S state ${ }^{5}$.

However, as was already noticed, the formal difference of two restricted states doesn't mean automatically that this states are physically different for $O$. That's the necessary but not sufficient condition, there should be also some effect available for $O$ observation that indicate their difference. For $\xi_{i}^{O}$ the only well-defined parameter is observable $Q_{O}$ value $q_{i}^{O}$, which is equal also to IP $J^{O}(n)$; it stipulates $\mathrm{BD}$ of $\xi_{1,2}^{O}$ for $O$. Hence one should explore whether some different effect can be attributed to $R_{O}$. The idea of this check is analogous to described above for D states. Suppose that $R_{O} \neq \xi_{i}^{O}$ for $O$ in BD sense, hence their relation should be expressed also by some $O$ internal parameter $g^{O}$ with the values $1 / 0$ correspondingly. But in QM such parameter should correspond to some $O$ observable, in particular, $R_{O}$ projector $P^{R}$ is the possible candidate. More generally because here three states are involved, it should be $O$ observable $G^{O}$, for which $R_{O}, \xi_{i}^{O}$ are its eigenstates: $G^{O} R_{O} \rightarrow g_{0}, G^{O} \xi_{i}^{O} \rightarrow g_{i}$, and $g_{0} \neq g_{i}$. But there is no $G^{O}$ with such properties, it follows from the fact that each $\xi_{i}^{O}$ has one and only projector $P_{i}^{O}=\xi_{i}^{O}$. Really from Spectral Theorem ${ }^{3}$ any $G^{O}$, for which $R_{O}, \xi_{i}^{O}$ are eigenstates, admits the decomposition:

$$
G^{O}=G^{\prime}+c_{0} P^{R}+c_{1} P_{1}^{O}+c_{2} P_{2}^{O}
$$

with $c_{i}$ - real, $G^{\prime}$ is an arbitrary operator for which $G^{\prime} P_{i}^{O}=0$, and $P^{R} P_{i}^{O}=0$. But $P_{i}^{O}$ 
constitute the complete algebra of projectors in $\mathcal{H}_{\mathcal{O}}$, i.e. $\sum P_{i}^{O}=I$, and so:

$$
P^{R}=P^{R} I=P^{R} \sum P_{i}^{O}=\sum P^{R} P_{i}^{O}=0
$$

Hence such $G^{O}$ doesn't exist and $R_{O}$ of (4) isn't proper ansatz for $\Psi_{M S}$ restriction. It indicates that to dispatch correct $\bar{Q}_{O}$ for pure MS ensemble, $O$ should observe the 'subjective' collapse of pure S state to one of $q_{i}^{O}$ at random with probability $P_{i}=\left|a_{i}\right|^{2}$, as follows from Graham-Hartle Theorem ${ }^{11}$. Below the mechanism of such collapse will be discussed in more detail.

Remind that in QM two kinds of uncertainties are presented: if the value $\tilde{g}$ of observable $G$ lays in the interval $g_{c} \leq \tilde{g} \leq g_{d}$, then depending on quantum state $\rho$, it can be either the stochastic value (SV) i.e. objectively $\tilde{g}=g_{i}$ with some probability $P_{i}^{\prime}$, or it can be truly uncertain (fuzzy) value (FV) $\tilde{g}$. The difference between this two cases revealed by $\bar{G}^{c}$ value for 'interference term' (IY) observable $G^{c}$ which indicates $\tilde{g}$ superposition. For MS it will be joint $\mathrm{S}, O$ observables, as the example, consider the symmetric MS IT:

$$
B=\left|O_{1}\right\rangle\left\langle O_{2}|| s_{1}\right\rangle\left\langle s_{2}\right|+j . c .
$$

Being measured by external $O^{\prime}$ via its interaction with S,O, it gives $\bar{B}=0$ for any MS mixture induced by $W^{s}$, but $\bar{B} \neq 0$ for pure MS states of (3). For example, for incoming S state $\psi_{s}^{s}$ with $a_{1,2}=\frac{1}{\sqrt{2}}, \Psi_{M S}$ is $B$ eigenstate with eigenvalue $b=1$. However $B$ value can't be directly measured by $O$ 'from insides ${ }^{5}$. The reason is that when $\mathrm{S}, O$ interaction finish, $\mathrm{S}$ become free particle and the joint S, $O$ observables aren't available for $O$, but only its own internal observables, as the restriction of MS to $O$ dictates. From the same reasons the whole set of nonlocal IT observables $\left\{B^{M S}\right\}$ is unavailable for $O$. Note that this difference between pure/mixed MS states is only statistical one, in particular, their distributions for $B$ (or other $B^{M S}$ ) overlap, because for $\bar{B}=0$ the probability $P_{B}\left(b_{1,2}\right)=.5$ for such mixture. Consequently even $O^{\prime}$ presumably can't discriminate pure/mixed S states in a single event, but only statistically for $N \rightarrow \infty$.

Let's compare $\Psi_{M S}$ restriction to $O$ denoted $\xi_{s}^{O}$ and the restriction for MS mixture - $R_{O}^{\text {mix }}$ obtained above. Consider $\xi_{s}^{O}$ first for incoming S state $\psi_{s}^{s}$, in $O^{\prime} \mathrm{RF}$ such $\Psi_{M S}$ is $B$ eigenstate with the eigenvalue $\tilde{b}=1$ - it's called here IT property; simultaneosly $q_{1}^{O} \leq \tilde{q}^{O} \leq q_{2}^{O}$ - the pointer property $(\mathrm{PP})$. Taken together this properties indicate that $\tilde{q}^{O}$ is located within the interval $\left\{q_{1}^{O}, q_{2}^{O}\right\}$, and so is principally uncertain inside it, i.e. this is $\tilde{q}^{O}$ superposition: $\tilde{q}^{O}=$ $q_{1}^{O}$.and. $\tilde{q}^{O}=q_{2}^{O}$, as $\tilde{b}=1$ value evidences for $O^{\prime}$. But $B$ is unavailable for $O$ and so $\xi_{s}^{O}$ can't posess IT property. Without it PP alone means only that for $\xi_{s}^{O} \tilde{q}^{O}$ is localized inside $\left\{q_{1}^{O}, q_{2}^{O}\right\}$ interval, but $\tilde{q}^{O}$ for $O$ can be either FV or SV within this limits. Meanwhile $R_{O}^{m i x}$ state has SV $q^{O}$ i.e. $\tilde{q}^{O}=q_{1}^{O}$. or. $\tilde{q}^{O}=q_{2}^{O}$, i.e. it also posess $\mathrm{PP}$, hence this property is identical for both regarded $O$ states. As was shown above any other $O$ observable also can't reveal $q^{O}$ superposition and $\xi_{s}^{O}$ distinction from $R_{O}^{m i x}$, so this states would be equivalent for $O$. Obviously $O$ can either distinguish $\xi_{s}^{O}, R_{O}^{m i x}$ via some effect, or fails to do it which seems to take place. Consequently $\Psi_{M S} \rightarrow \xi_{s}^{O}$ restriction can be formally written as:

$$
\xi_{s}^{O}=R_{O}^{m i x}=\xi_{1}^{O} \cdot o r \cdot \xi_{2}^{O}
$$

It means that for $\Psi_{M S}$ in each event $\xi_{1}^{O}$ or $\xi_{2}^{O}$ appears for $O$ at random, Consequently $\Psi_{M S} \rightarrow \xi_{s}^{O}$ inference map $M_{O}$ is stochastic and results in the subjective state collapse observed by $O$. To reproduce the correct expectation values $\bar{Q}_{O}^{l}$ for arbitrary $l$, the corresponding $\xi_{i}^{O}$ probabilities are $P_{i}=.5$. The same arguments are applicable for $\Psi_{M S}$ of (3) with arbitrary $a_{i}$, it results in the same solution (9) for $\xi_{s}^{O}$, the resulting $\xi_{i}^{O}$ ensemble is stochastic with probabilities $P_{i}=\left|a_{i}\right|^{2}$. 
It's easy to see that D, $O$ decoherence ${ }^{1,2}$ by their environment doesn't play any principal role in our theory, rather it can only amplify and stabilize the described mechanism. We conclude that EA together with obtained restriction $\mathrm{MS} \rightarrow O$ permit to construct the formalism of quantum measurements without QM Reduction Postulate. As was shown the main source of stochasticity is the principal loss of specific information in $\mathrm{S} \rightarrow O$ information channel. This information characterizes the purity of $\mathrm{S}$ state $^{8}$, as the result $O$ can't discriminate the pure/mixed $\mathrm{S}$ states. The most interesting feature of this formalism is that the same $\mathrm{S}$ state can be stochastic in $O$ $\mathrm{RF}$, but evolve linearily in $O^{\prime} \mathrm{RF}$. The detailed explanation of this effect is given in Algebraic Formalism $^{8}$, here we notice only that $O$ and $O^{\prime}$ deal with different sets of MS observables, and so the transformation of MS state between them can't be unitary. Obtained results agree completely with our calculation in $C^{*}$ Algebras formalism ${ }^{8}$, in this case the inference map is the restriction of MS observable algebra to $O$ (sub)algebra.

\section{References}

[1] P.Busch, P.Lahti, P.Mittelstaedt, Quantum Theory of Measurements, (Springer-Verlag, Berlin,1996), pp. 8-26

[2] W.D'Espagnat Found Phys. 20 (1990) 1157

[3] J.M.Jauch 'Foundations of Quantum Mechanics' (Adison-Wesly, Reading, 1968), pp. 85-116

[4] (1972) G.Emch, 'Algebraic Methods in Statistical Physics and Quantum Mechanics', (Wiley,N-Y,1972)

[5] T.Breuer Synthese 107 (1996) 1

[6] P.Mittelstaedt 'Interpretation of Quantum Mechanics and Quantum Measurement Problem', (Oxford Press, Oxford,1998) pp. 67-109

[7] K.Svozil 'Randomness and undecidability in Physics', (World Scientific, Singapour,1993) pp. 46-87

[8] S.Mayburov, Information-Theoretical Restrictions in Quantum Measurements, quantph/0506065; Int. J.Theor. Phys, (to appear)

[9] E.Wigner, 'Scientist speculates',(Heinemann, London,1961), pp 47-59

[10] W.Zurek Phys. Rev. D26 (1982) 1862

[11] J.B.Hartle Amer. J. Phys. 36 (1968) 704 